\author{
بررسى مقادير درجه روز --رشد مراحل فنولوزيك در تعدادى از ارقام زراعى إئ

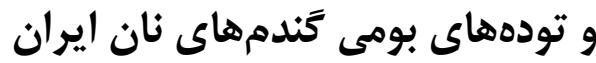 \\ هادى على يور '، حسين عبدى 'و محمدرضا بىهمتا؟ \\ ا- استاديار گروه توليد و زنتيك گياهى، دانشخاه اروميه، اروميه، (نويسنده مسوول:

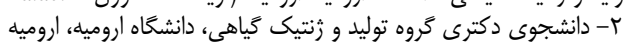

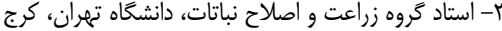 \\ تاريخ ارسال: • •

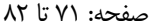

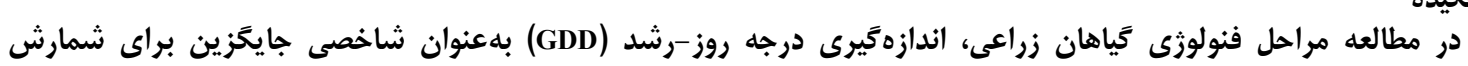

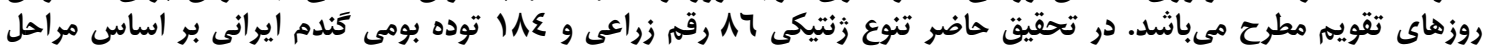

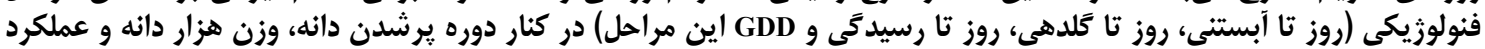

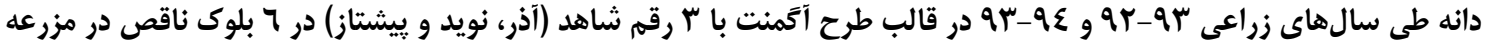

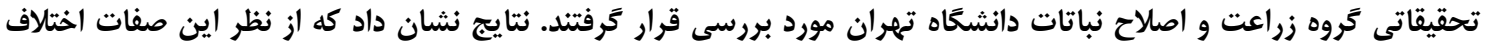

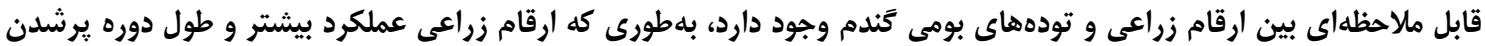

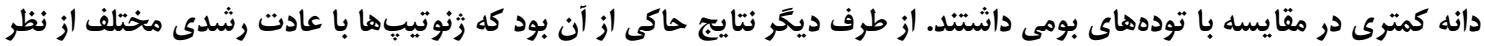

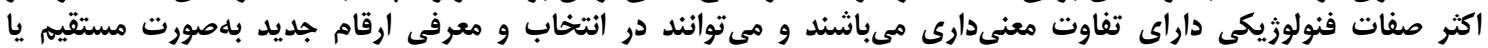

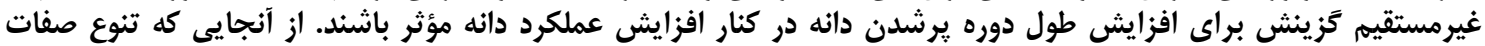

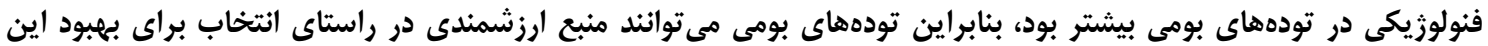

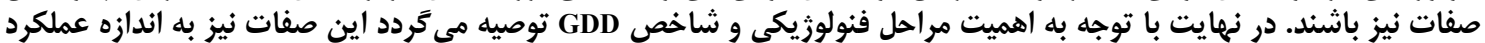

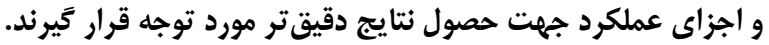

وازههاى كليدى: ارقام زراعى، تنوع زنتيكى، تودههاى بومى، درجه روز -رشد، نياز حرارتى

شد متتواند جايكزين شمارش روزهاى تقويم باشد. اين إين

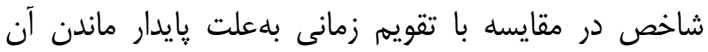

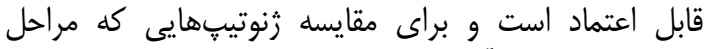

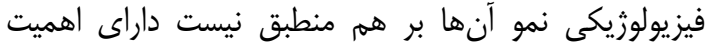

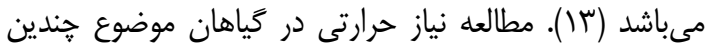

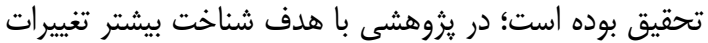

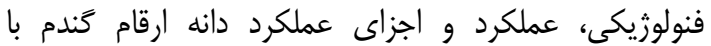

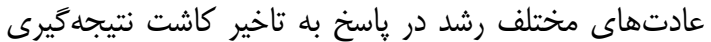

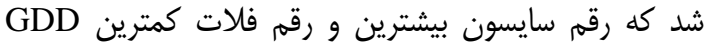

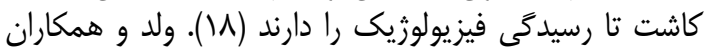

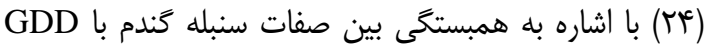
بر اهميت اين شاخص تأكيد داشتند. اما نادرى (هD (1) كزارش

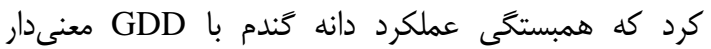

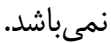
با توجه به تنوع جغرافيايى وسيع در نواحى مختلف كشورو، فئورئ

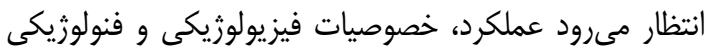

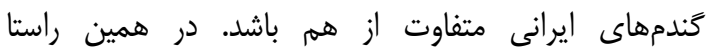

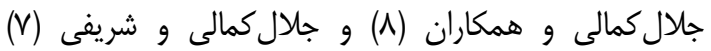

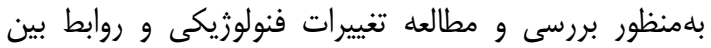

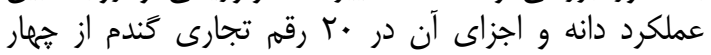

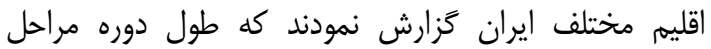

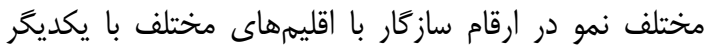

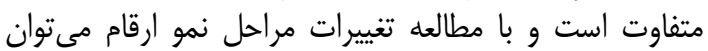
تغييرات عملكرد دانه را توضيح داد. يوسفى مقدم و همكاران
مقدمه

كندم (Triticum aestivum L.) مهمترين غله در سطح

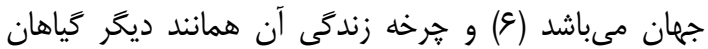

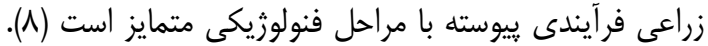

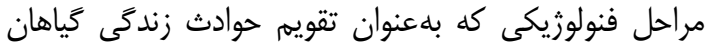

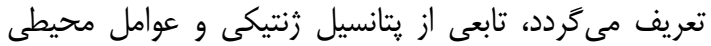

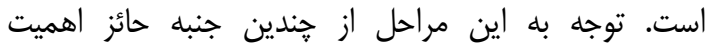

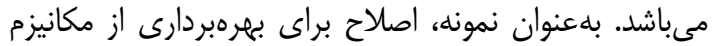

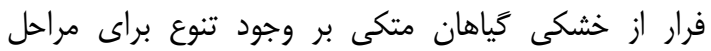

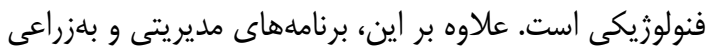

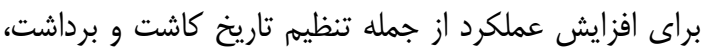

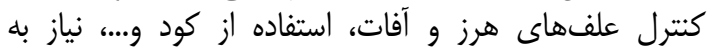

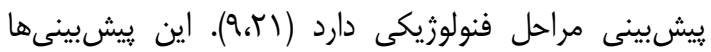

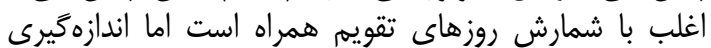

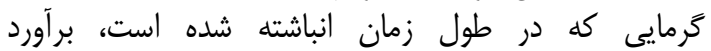

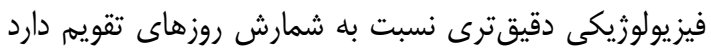

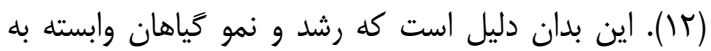

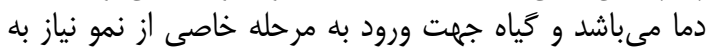

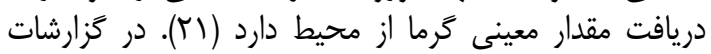

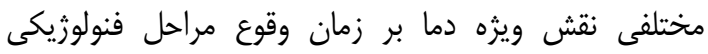

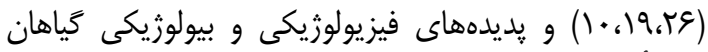

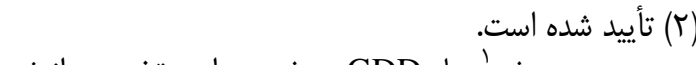

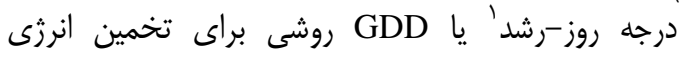
حرارتى گياه براى تكميل مراحل تكوينى است و حنانجه اشاره 
در ترسيم دندركرام تجزيه خوشهاى از فاصله اقليدسى و روش Ward استفاده شد.

\section{نتايج و بحث}

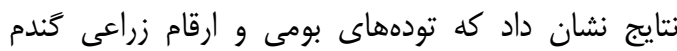

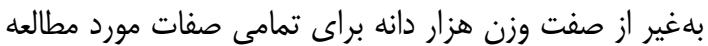

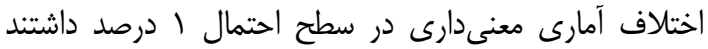

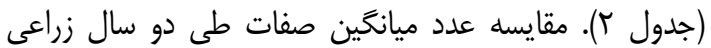

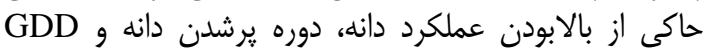

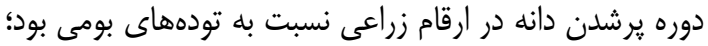

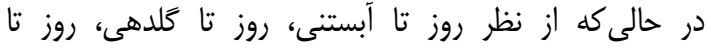

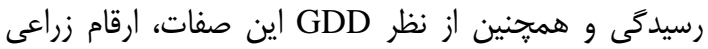

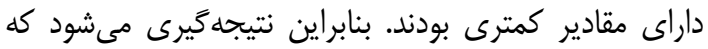

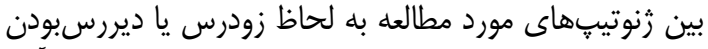

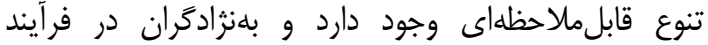

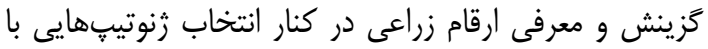

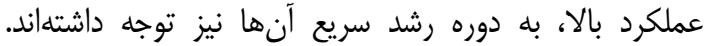

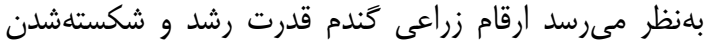

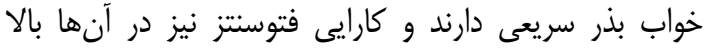

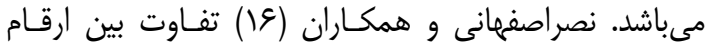

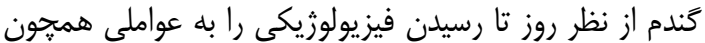

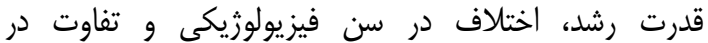

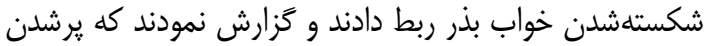

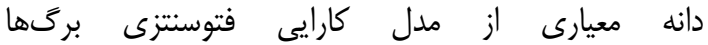

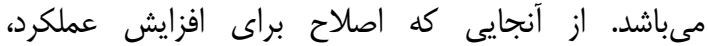

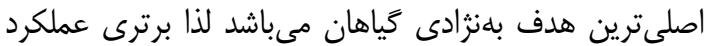

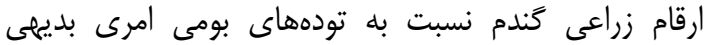

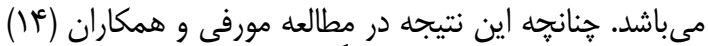

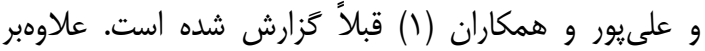

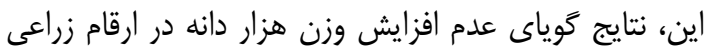

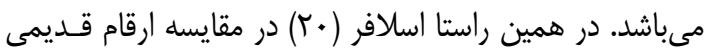

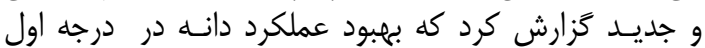

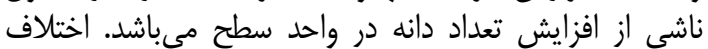

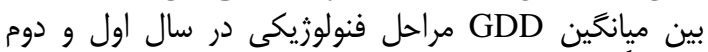

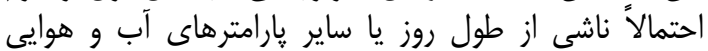

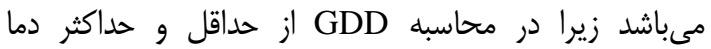

نتايج تجزيه واريانس ارقام شاهد در طرح آَحمنت به تفكيك استفاده شده است.

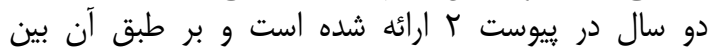

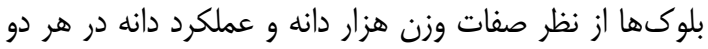

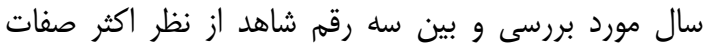

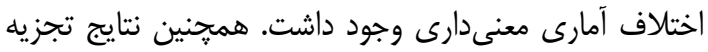

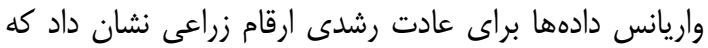

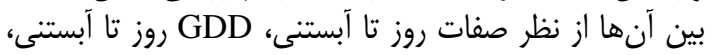

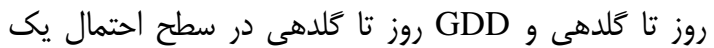

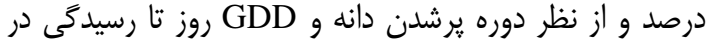

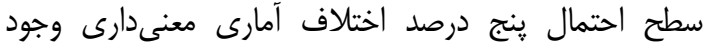
داشت (جدول rا). ارقام زراعى با عادت رشدى إِاييزه بهجز

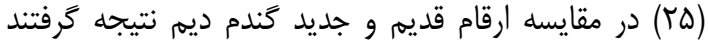

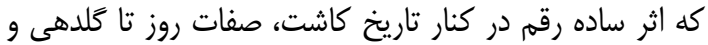

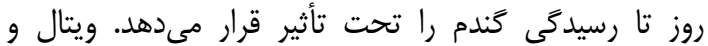

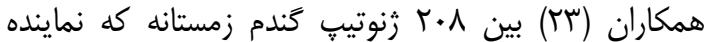

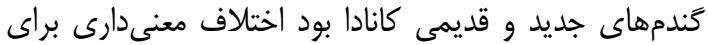

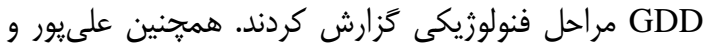

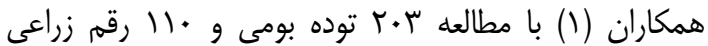

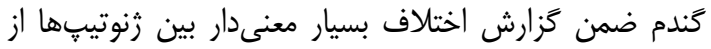

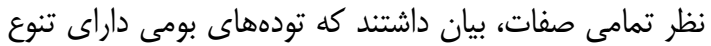

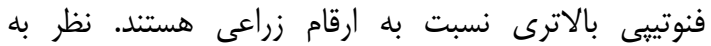

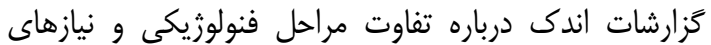

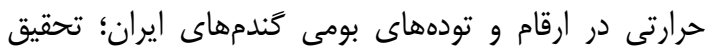

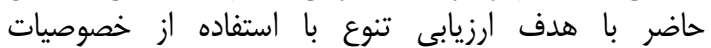

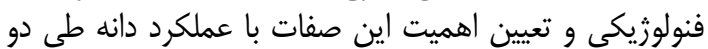

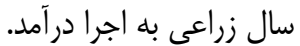

\section{مواد و روشها}

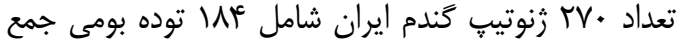

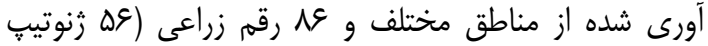

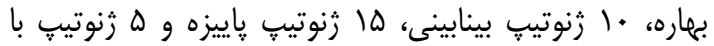

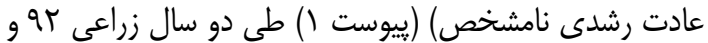

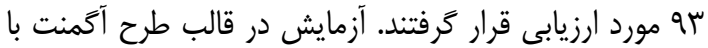

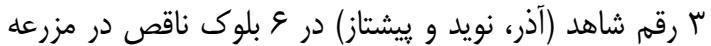

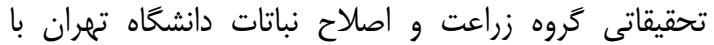

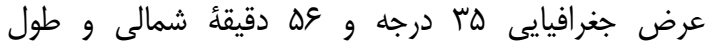

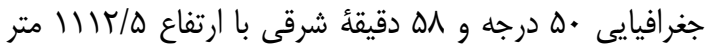

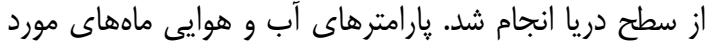

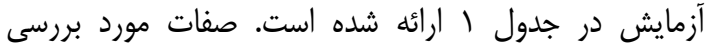

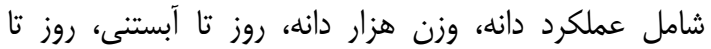

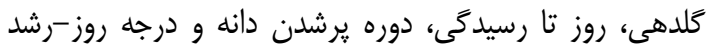

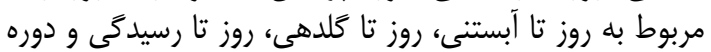
ير ترشدن دانه بودند. درجه روز رشد بر اساس اطلاعات هواشناسى و با احتساب

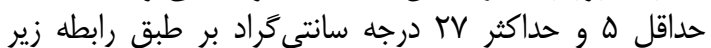
$\mathrm{GDD}=\sum_{1}^{\mathrm{n}} \frac{\mathrm{T}_{\text {max }}+\mathrm{T}_{\text {min }}}{2}-\mathrm{T}_{\text {base }}$

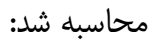
كه در اين معادله Th: دماى روزانه،

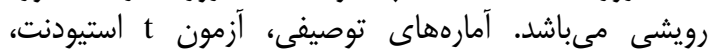

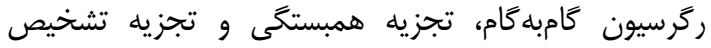

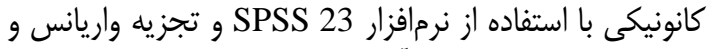

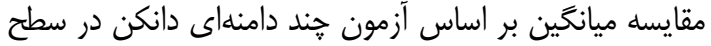

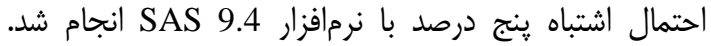

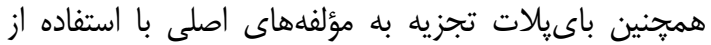

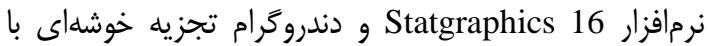

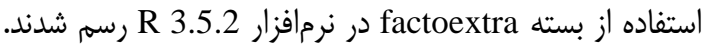


همكاران (^) كزارش كردند كه رقم شهريار -از ارقام زمستانه-

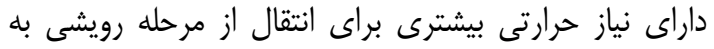

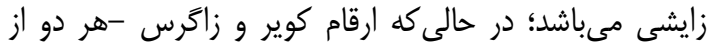

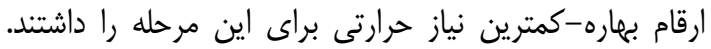

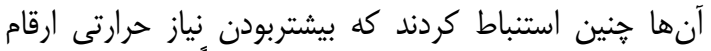

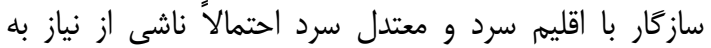

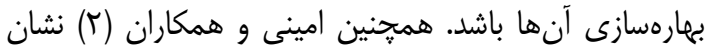

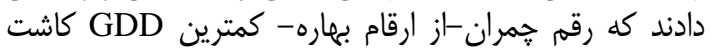
تا رسيدگى كامل را دارد.
دوره برشدن دانه براى باقى صفاتى كه ميانگين مربعات آنها

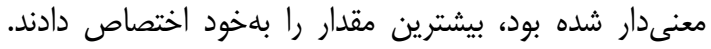

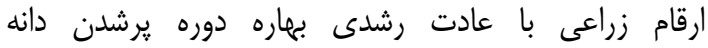

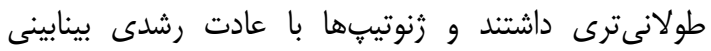

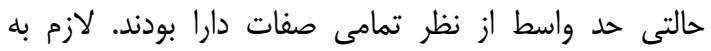

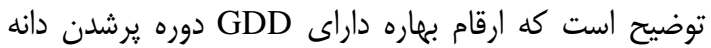

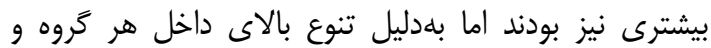

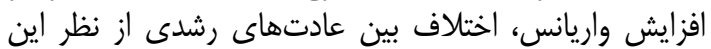

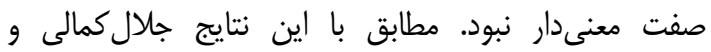

Table 1. Monthly climatic parameters during two crop seasons

جدول ا- يارامترهاى آب و هوايى ماههاى اجراى آزمايش طى دو سال زراعى

\begin{tabular}{|c|c|c|c|c|c|c|c|}
\hline خرداد & ارديبهشت & فروردين & اسفند & بهمن & s & آذر & \\
\hline & & & & & & & سال اول \\
\hline . $/ 4 t$ &.$/ 90$ & $1 / 49$ & $V / \Delta V$ & . & $\cdot / \cdot V$ & $1 / r$ & متوسط بارندگى (mm) \\
\hline $19 / 0$ & $1 \% \%$ & $s / r q$ & $r / v q$ & $-1 / V$ & $-r / r$ & I/DT & حداقل درجه حرارت (م) \\
\hline$T F / V$ & $r \cdot / 9$ & $1 \pi / 1$ & १/१५ & $r / \mu$ & $1 / 4 T$ & $\Delta / A V$ & متوسط درجه حرارت (C'C) \\
\hline \multirow[t]{2}{*}{ ( } & $T V / G$ & $19 / \Delta$ & $\mid \omega / \kappa^{c}$ & $N / D S$ & $\Delta / 9 \Lambda$ & 1.11 & حداكثر درجه حرارت (C) \\
\hline & & & & & & & سال دوم \\
\hline$\cdot / K \Delta$ &.$/ \cdot V$ & $1 / / V$ & T & $\cdot / \Delta S$ & $\cdot / T \Delta$ & $\cdot / A V$ & متوسط بارندگى (mm) \\
\hline $\mathrm{IV} / \mathrm{V}$ & $1 \pi / r$ & $\mathrm{~V} / \Lambda \mathrm{F}$ &.$/ 4 q$ & $r / \mu$ & .1 .0 & $r / \Delta$ & حداقل درجه حرارت (م) \\
\hline re/T & $r \cdot / r$ & $1 \% / \Lambda$ & $s / V^{\mu}$ & $\mathrm{V} / \mathrm{IT}$ & $\Delta / \backslash \Lambda$ & $8 / \cdot 1$ & متوسط درجه حرارت (C'C) \\
\hline$r y /$. & $\mathrm{TV} / \mathrm{q}$ & $19 / \pi$ & $1 \% / \mu$ & $\mid r / 1$ & $1 . / 4$ & 1.10 & 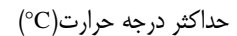 \\
\hline
\end{tabular}

\section{تجزيه ركرسيون}

هنخامى كه تعداد متغيرهان مان مستقل موثر بر صفت وابسته

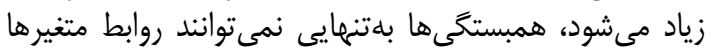

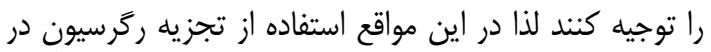

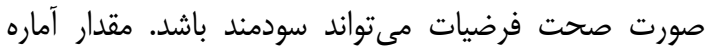

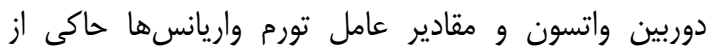

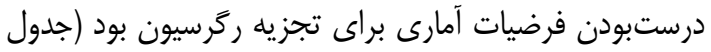

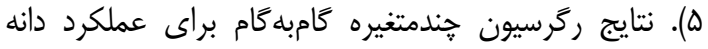

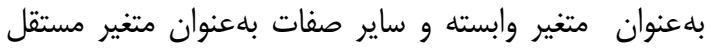

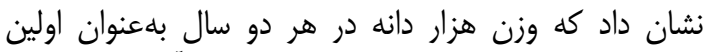

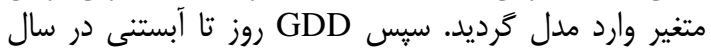

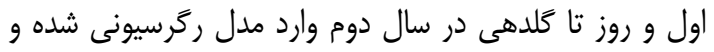

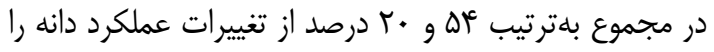

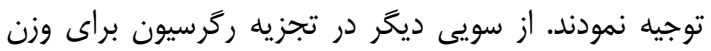

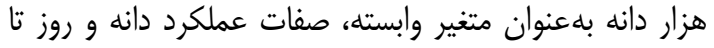

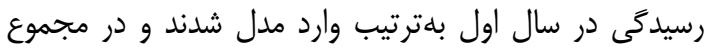

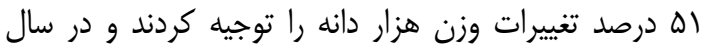

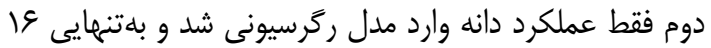

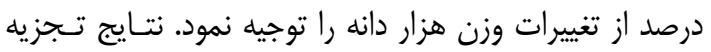

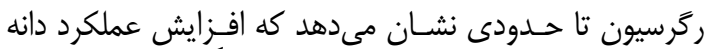

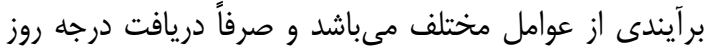

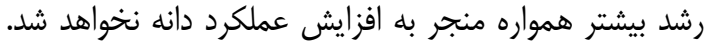

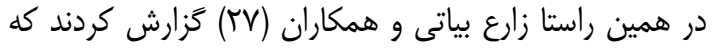

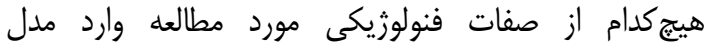

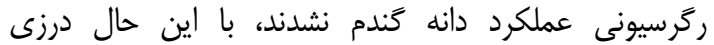

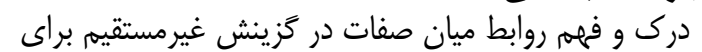

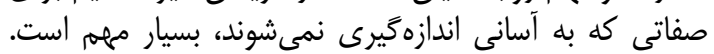

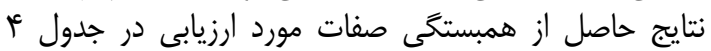

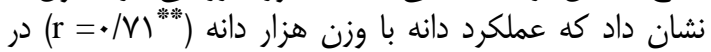

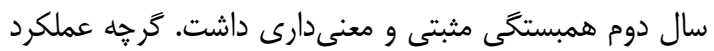

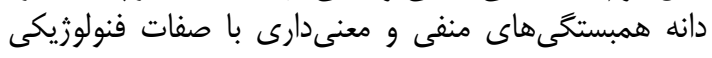

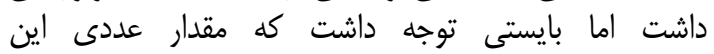

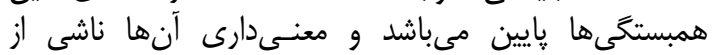

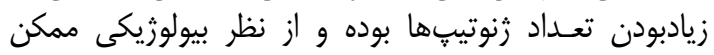

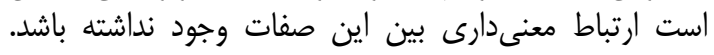

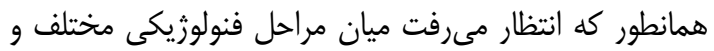

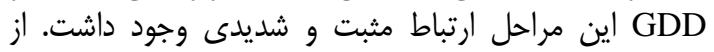

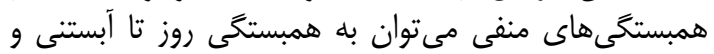

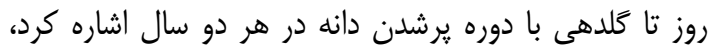

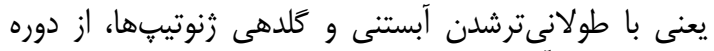

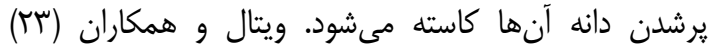

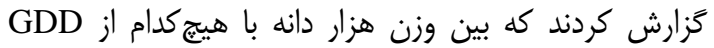

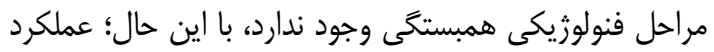

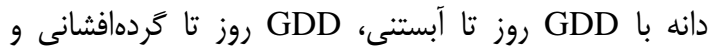

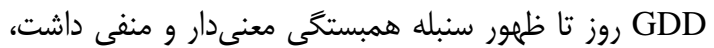
اما بين عملكرد و GDD روز تا رسيدگى رو رابطه خطى رئى مشاهده

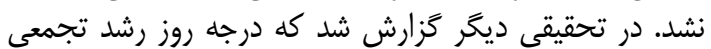

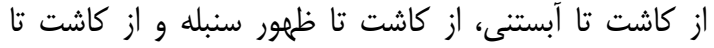

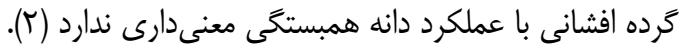


متغيرى مىباشد كه بلتنهايى بيش از سب درصد از تغييرات

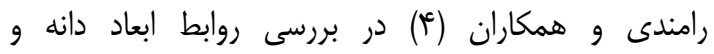
عملكرد دانه را توجيه كرد.

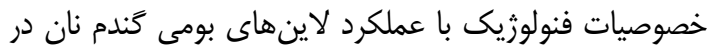

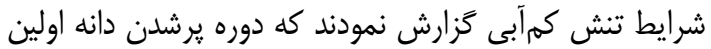

جدول r- آمارههاى توصيفى صفات مورد بررسى و آزمونهاى t - استويدنت مربوطه Table 2. Descriptive statistics of investigated traits and the relevant t-student tests

\begin{tabular}{|c|c|c|c|c|c|c|c|c|c|c|c|c|}
\hline \multirow{2}{*}{ سالها t- جفتى براى } & \multicolumn{2}{|c|}{ t- مستقل } & \multicolumn{2}{|c|}{ انحراف معيار } & \multicolumn{2}{|c|}{ ميانغين } & \multicolumn{2}{|c|}{ حداكثر } & \multicolumn{2}{|c|}{ حداقل } & \multirow[b]{2}{*}{ جمعيت } & \multirow[b]{2}{*}{ صفات } \\
\hline & سال دوم & سال اول & سال دوم & سال & سور & سال & دول & سال & دور & سال & & \\
\hline$-\Gamma / \Gamma \Lambda^{*}$ & & & $1 / 10$ & .199 & $T / \uparrow \wedge$ & $T / M F$ & $\mathrm{~V} / \mathrm{I}$ & $0 / 48$ &.$/ T \Lambda$ &.$/ \mu \Lambda$ & تودهoا & \\
\hline$-1 / \wedge \Delta^{\mathrm{ns}}$ & $-\Gamma / \Delta \gamma^{* * *}$ & $-\varphi / / r^{*}$ &.$/ 19$ &.$/ 91$ & $r / 91$ & $r / V \Delta$ & $\Delta / \Delta$ & $\Delta / \AA \wedge$ &.$/ 199$ & תוq/. & ارقام & GY \\
\hline$-1 \Psi / \digamma^{\text {米綃 }}$ & & & $1 \cdot / V$ & $V / V I$ & $F r / \Delta$ & $r . / 9$ & $q \pi / \Gamma$ & $\Delta T / T$ & $1 / 99$ & $I T / S$ & تودهoها & \\
\hline$-1.19^{* * *}$ & $-\cdot / \Delta q^{n s}$ & $-\cdot / r T^{n s}$ & $V / q C^{c}$ & SIAV & $\kappa \mu / T$ & $\mu / / r$ & $9 D / \Lambda$ & $\uparrow V / \Delta$ & $T r / r$ & $1 \% / \Delta$ & ارقام & TGW \\
\hline$-\varphi / r^{*}{ }^{* * *}$ & & & 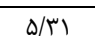 & F/q9 & IVD & IVte & 191 & Mr & $\mid \Delta F^{\circ}$ & IQT & تودهها & \\
\hline - & १/१. & $11 / r^{* * *}$ & s/4 & $81 \cdot 1$ & $19 \mathrm{~V}$ & 199 & 19. & Mr & $1 D S$ & $1 \Delta T$ & ارقام & DB \\
\hline 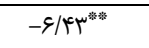 & & & $r / q V$ & $r / 19$ & $1 \Lambda$. & IVA & 194 & 119 & r & 191 & تودهها & \\
\hline 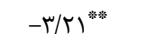 & $1 \cdot / /^{\mu * w}$ & $9 / 9 V^{* * m}$ & D/r & $r / q V$ & IVr & IVT & 190 & IAV & 194 & 191 & ارقام & DF \\
\hline$-9 / 9)^{\text {糙 }}$ & & & $r / q \Lambda$ & $\Gamma / 90$ & TIS & זוץ & Trg & MrI & $r \cdot 1$ & $r \cdot \Delta$ & تودهها & \\
\hline$-ه / 9 \uparrow^{* * *: 5}$ & 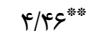 & $\Gamma / \Gamma \varphi^{* * *}$ & $r / v$. & $r / v \varepsilon$ & זוץ & rII & rrq & TrI & $r \cdot \Lambda$ & $r . q$ & ارقام & DM \\
\hline 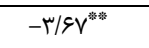 & & & $r / T \Delta$ & $\Gamma / \backslash Q$ & $r \Delta / \bar{s}$ & 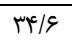 & 19 & 19 & tr & TE & تودهها & \\
\hline$-r / \Delta \gamma^{* * *}$ & $-\Lambda / \Lambda Y^{* *}$ & $-V / \uparrow 8^{*}$ & $r / v \Delta$ & r/l & $f \cdot / c$ & rN/G & 19 & $\Delta T$ & r. & re & ارقام & GF \\
\hline$r \cdot / \Lambda^{* * * *}$ & & & 111 & $9 \mathrm{~V} / 9$ & IFTY & IQTA & IVWV & שוVו & $1 . \mu r$ & live & تودهها & \\
\hline $1 \Gamma / 8^{* * *}$ & 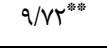 & $11 / *^{* * * 6}$ & 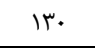 & 111 & ITVA & $|r \Lambda|$ & $I V \Delta D$ & IVIT & 1.94 & live & ارقام & GDDB \\
\hline $10 / \omega^{* \omega *}$ & & & $1 \cdot V$ & $99 / \%$ & IDF. & IGTr & ג & १یrq & 1194 & ITAD & تودهها & \\
\hline $19 / 0^{* * *}$ & $1 \cdot / r^{* * * *}$ & $9 / \mathrm{V} \wedge^{* * *}$ & $11 \pi$ & $99 / 9$ & Irate & 1998 & $|1 \times|$ & 11.1 & ITIK & ITAD & ارقام & GDDF \\
\hline $\mathrm{Q} / \Delta \Lambda^{* * *}$ & & & $9 T / \pi$ & 1.1 & rTDS & TEYTE & אואז & TEY. & $r \mid \Delta \Lambda$ & TrIV & تودهها & \\
\hline$\Delta / V^{e^{*}}$ & 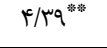 & $\varphi / \mu^{\mu^{*}}$ & $9 \% / 9$ & $9 \Delta / \Delta$ & r.r & req & $T V \cdot r$ & זqY. & rIQA & Trefe & ارقام & GDDM \\
\hline$-r / r q^{*}$ & & & $9 D / T$ & $91 / 0$ & AIV & $1 \cdot 1$ & $111 \%$ & זrו1 & $\Delta \cdot \Delta$ & 9.9 & تودهها & \\
\hline$-r / 11^{*}$ & $-V / \wedge \Delta^{* * *}$ & $-g / 1 \Gamma^{* * * a n}$ & $v a / v$ & $M / r$ & 9.9 & 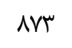 & $1.9 V$ & אזוו & gAT & $\Delta \& q$ & ارقام & GDDG \\
\hline
\end{tabular}

جدول س- تجزيه واريانس و مقايسه ميانخين زنوتيبها از نظر عادت رشدى

Table 3. Analysis of variance and means comparison of studied genotypes based on growth habit

\begin{tabular}{|c|c|c|c|c|c|c|c|c|c|c|c|}
\hline & & & & & & & & \multicolumn{2}{|c|}{ ميانغين مربعات } & \multirow{2}{*}{ آزادى } & \multirow{2}{*}{ منابع تغييرات } \\
\hline GDDG & GDDM & GDDF & GDDB & GF & DM & DF & DB & TGW & GY & & \\
\hline $994 q^{\text {ns }}$ & $|V Q 9|^{*}$ & 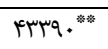 & qTrQ.*** & $r q / q^{*}$ & $r q / \Lambda^{\mathrm{nS}}$ & $1 \xi^{* * * 6}$ & $T \varphi^{* \text { **: }}$ & $s / k^{\mathrm{ns}}$ & $\cdot /^{\mathrm{ns}}$ & $r$ & عادت رشدى \\
\hline \multirow[t]{2}{*}{ rq. } & $\Delta V \& N / V$ & Q४VQ/ & $\| 114$ & $9 / V$ & $N / q$ & $r \mu / 1$ & rו/ & צחוז &.$/ 4 \lambda$ & $\mathrm{v \Lambda}$ & خطا \\
\hline & & & & & & & & & & \multicolumn{2}{|c|}{ مقايسات ميانكَين } \\
\hline$\wedge \& 1 / 1$ & $r r V / /^{a}$ & $|\Delta| \varepsilon \mid \cdot^{a}$ & IFtr/Fa & $r v / v^{b}$ & $r / r / \Lambda$ & $1 V q^{a}$ & $\mid V^{a}$ & ل & $r / A r$ & & بإبيزه \\
\hline$\wedge \vee \& / q$ & $r \mu r \Delta / q^{a b}$ & $|+\& 9 /|^{a b}$ & |rgr/g/g & $\Gamma / q^{a b}$ & $r / r / \Delta$ & $\mid y e^{a b}$ & $19 \Lambda^{a b}$ & $r V / r$ & $r / 99$ & & بينابينى \\
\hline $9 . . / 1$ & rMrr/q & IFtr/A & $1 r^{m} \cdot 1 / \kappa^{b}$ & $\mathrm{r} .1 .^{\mathrm{a}}$ & $r I I / V$ & $\mid v^{b}$ & $19 \Delta^{b}$ & $r V / \Delta$ & $r / M$ & & بهاره \\
\hline
\end{tabular}

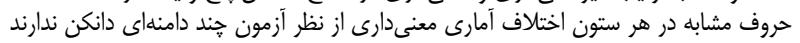

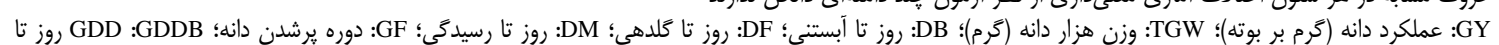

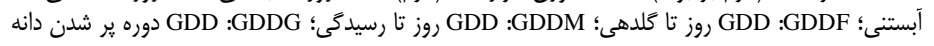


جدول عا- نتايج همبستگى بين صفات مورد ارزيابى (عناصر پايين قطر اصلى مربوط به سال اول و عناصر بالاى قطر اصلى مربوط

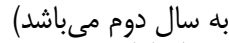

Table 4. Correlation results among investigated traits (upper diagonal is related to first year and below diagonal is related to second year)

\begin{tabular}{|c|c|c|c|c|c|c|c|c|c|c|}
\hline GDDG & GDDM & GDDF & GDDB & GF & $\mathrm{DM}$ & DF & DB & TGW & GY & صفات \\
\hline 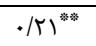 & $-\cdot 1 \cdot \wedge^{\mathrm{nS}}$ & $-\cdot / r r^{\text {絭 }}$ & $-+/ r^{\text {絭畨 }}$ & . $/ r^{* * * 0 \%}$ & $-\cdot \cdot \cdot \wedge^{\mathrm{ns}}$ & $-\cdot / r^{\text {券旁 }}$ & 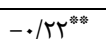 & $\cdot / 4 .^{* * \pi}$ & $1 / \cdots$ & GY \\
\hline$\cdot / r^{*}$ & $\cdot / \cdot 1^{\mathrm{ns}}$ & $-\cdot 1 \cdot 9^{\mathrm{ns}}$ & $-\cdot 1 \cdot 9^{\mathrm{ns}}$ & $\cdot / 11^{\mathrm{ns}}$ & $\cdot / \cdot 1^{\mathrm{ns}}$ & $-\cdot 1 \cdot 9^{\mathrm{ns}}$ & $-\cdot / \cdot \wedge^{\mathrm{ns}}$ & $1 / \cdot$ & $\cdot|r|^{* *}$ & TGW \\
\hline.$- \cdot 19 V^{\text {管 }}$ & $\cdot|q|^{\mu^{*} \cdot \%}$ & $1 / \ldots{ }^{\text {蜳 }}$ & $\cdot / 9 \Lambda^{\text {券菏 }}$ & $-\cdot / \mathrm{V}^{\text {粦菂 }}$ & 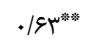 & $1 / \cdots$ & 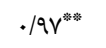 & $-\cdot 1 \cdot 9^{\mathrm{ns}}$ & 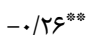 & DF \\
\hline.$/ / f^{* *}$ & 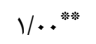 & 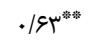 & 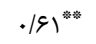 & $-\cdot \cdot \cdot 1^{\mathrm{ns}}$ & $1 /$. & 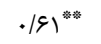 & $\cdot / \Delta \Lambda^{\text {**ak }}$ & $\cdot / \cdot r^{\mathrm{ns}}$ & $-\cdot / 1 \mu^{* *}$ & DM \\
\hline$-.199^{*{ }^{*}}$ & $\cdot \mid q r^{* * *}$ & $\cdot / 9 V^{\text {***as }}$ & $1 / \cdot$ & 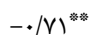 & $\cdot 109^{\circ * 0.0}$ & $\cdot 19 \Lambda^{* * *}$ & 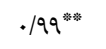 & $-\cdot / 1 \cdot{ }^{\mathrm{ns}}$ & $-\cdot / T V^{\text {㭗承 }}$ & GDDB \\
\hline 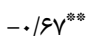 & 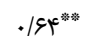 & $1 /$. & 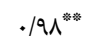 & $-\cdot / V)^{\text {*t* }}$ &.$|9|^{\text {緔 }}$ &.$/ 99^{\text {***as }}$ & 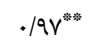 & $-\cdot \cdot \cdot 1^{\mathrm{ns}}$ & 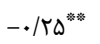 & GDDF \\
\hline.$/ 1 f^{*}$ & $1 / \cdot$ & $\cdot|9|^{* * *}$ & $\cdot / \Delta \Lambda^{* * * w^{*}}$ & $\cdot / 11^{\mathrm{ns}}$ & $1 / .^{*{ }^{*}}$ & $\cdot|8|^{* * * 3}$ & $\cdot / \Delta \Lambda^{* w^{*}}$ & $\cdot / \cdot r^{\mathrm{ns}}$ & $-\cdot / 1 r^{*}$ & GDDM \\
\hline $1 /$. & 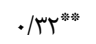 & $-\cdot / \Delta \Delta^{* * \omega^{*}}$ & $-\cdot / \Delta \Delta^{\text {**an }}$ & $.191^{* * *}$ & 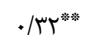 & $-\cdot / \Delta \Delta^{*}$ & 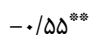 &.$/ M r^{*}$ & 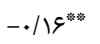 & GDDG \\
\hline
\end{tabular}

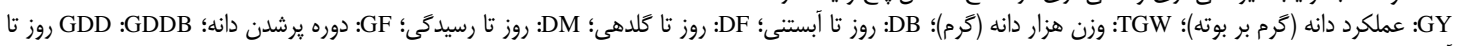

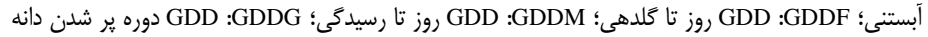

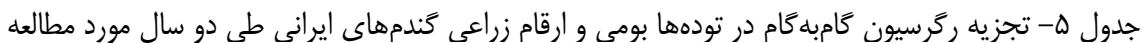

Table 5. Stepwise regression analysis in Iranian wheat landraces and cultivars during the two studied cropping seasons

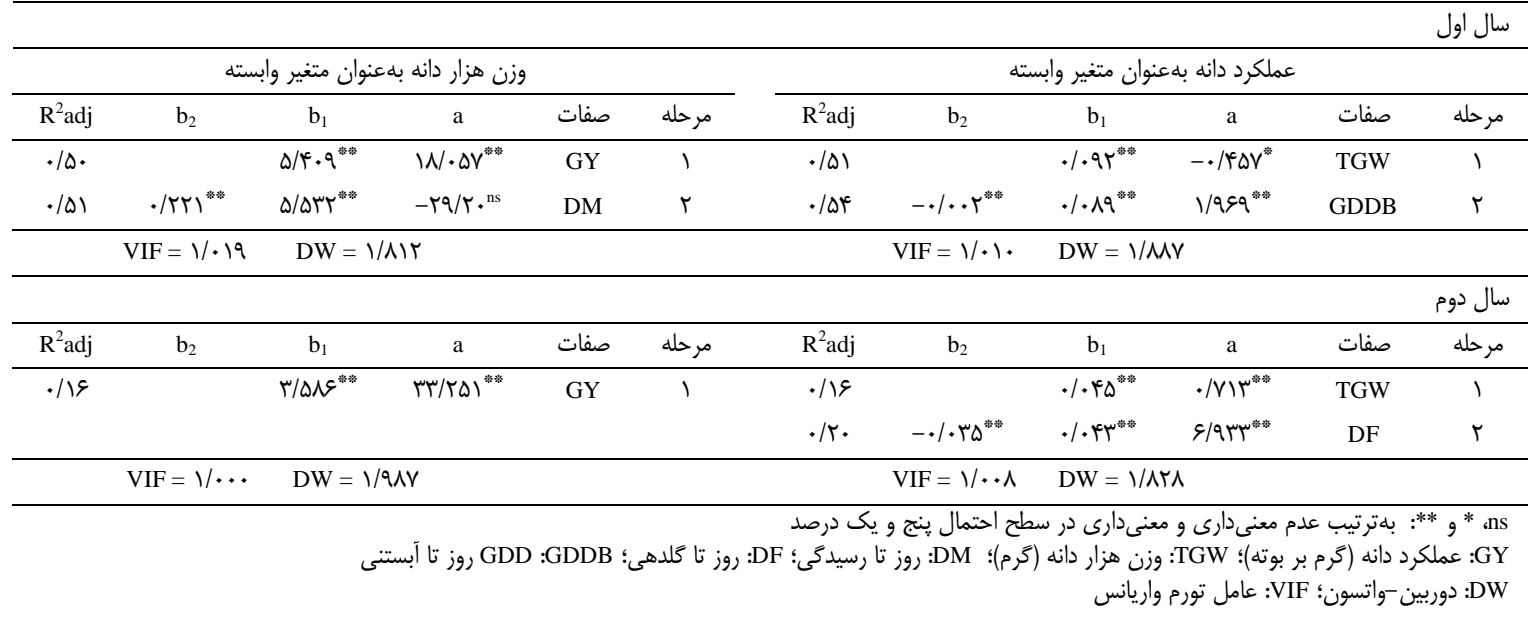

دوره بِشدن دانه كمتر از •و درجه، با روز تا رسيدگى تقريباً

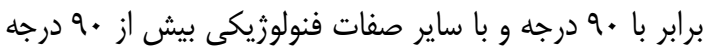

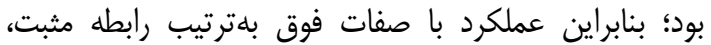

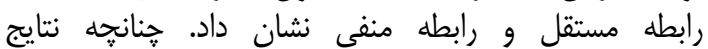
همبستخى ييرسون نيز مويد اين مطلب بودئ تجزيه خوشهاي

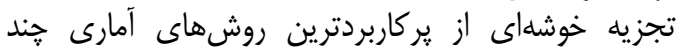

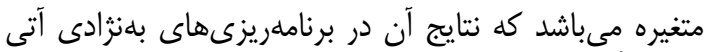

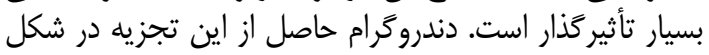

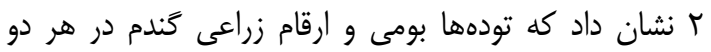

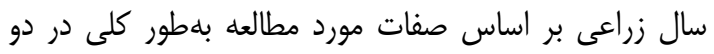

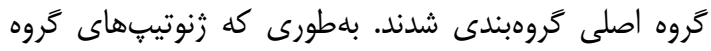

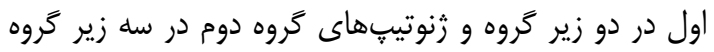

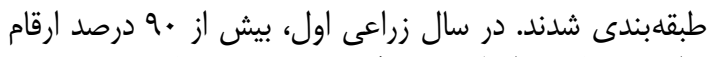

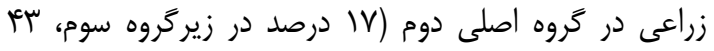

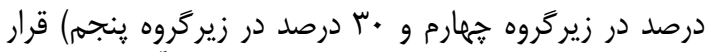

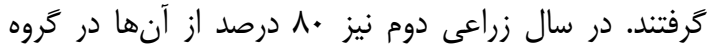

\section{تجزيه به مؤلفههاى اصلى}

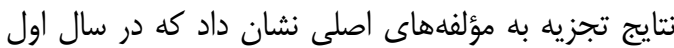

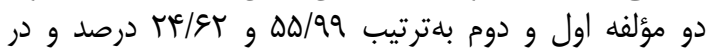

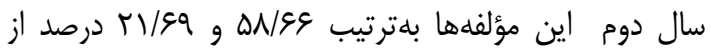

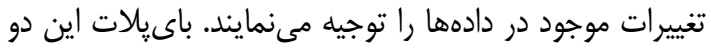

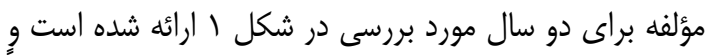

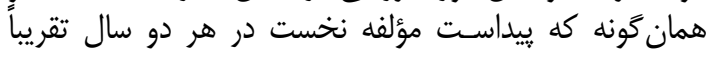

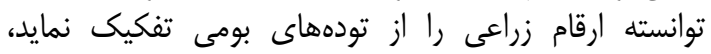

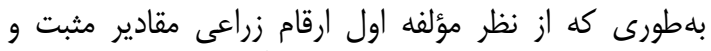

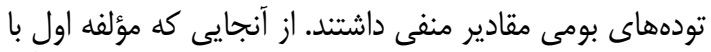

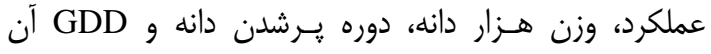

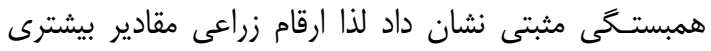

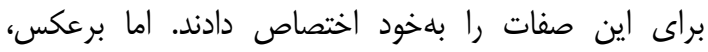

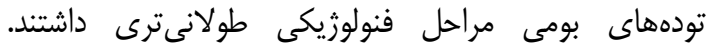

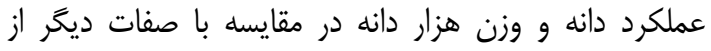

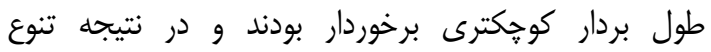
هايينترى داشتند و نظر به اينكه زاويه بردار اين صفات بار بار 
تابع تشخيص از ديكر روشهاى تاييد كرومبندى مىباشد.

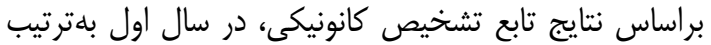

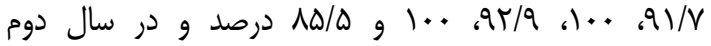

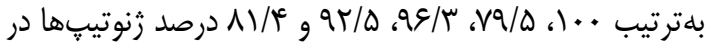

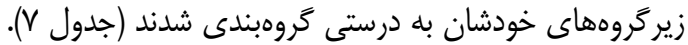

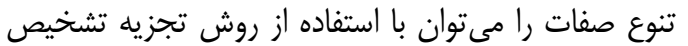

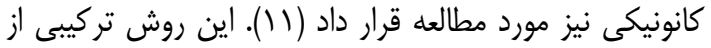

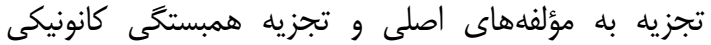

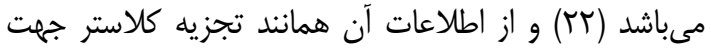

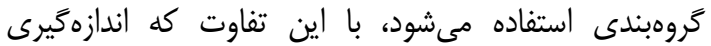

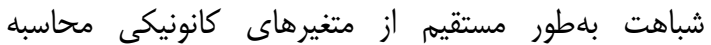

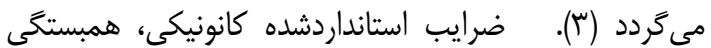

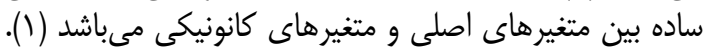

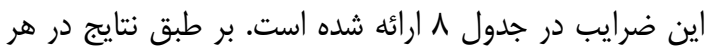

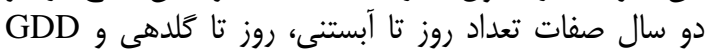

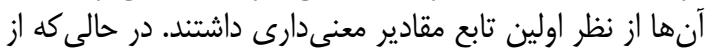

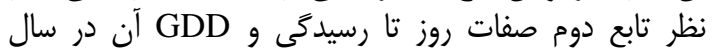

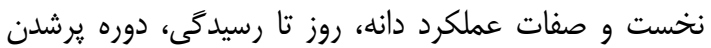

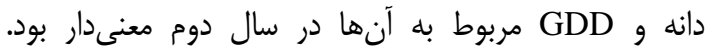

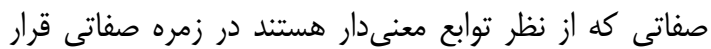

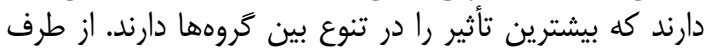

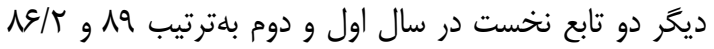
درصد از تغييرات موجود در دادهها را توجيه كردند.

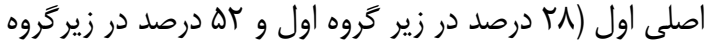

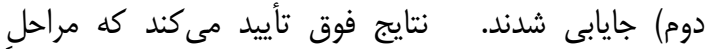

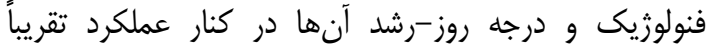

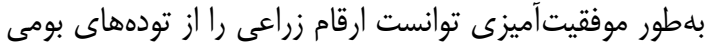

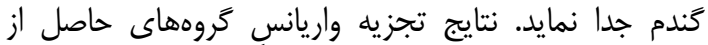

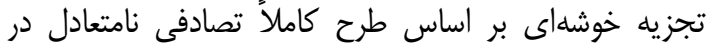

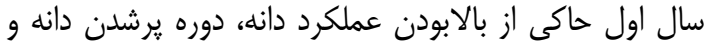

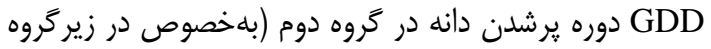

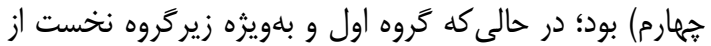

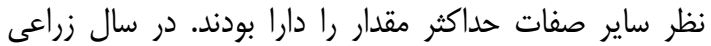

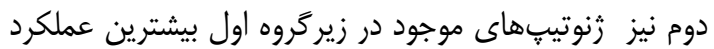

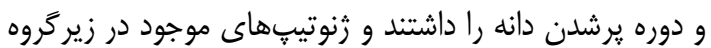

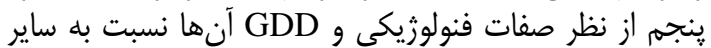

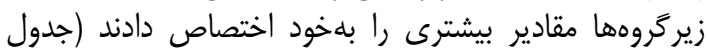

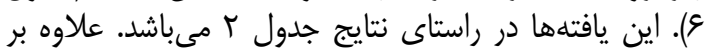

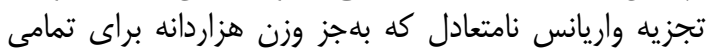

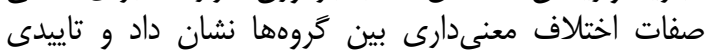

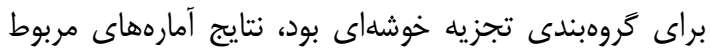

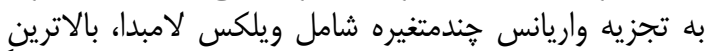

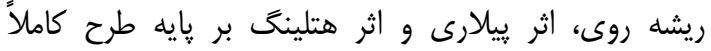

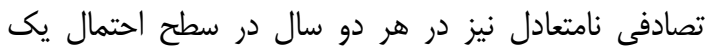

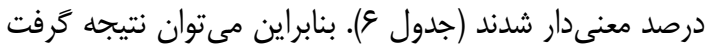

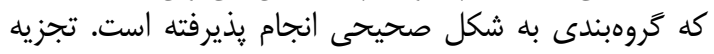

الف

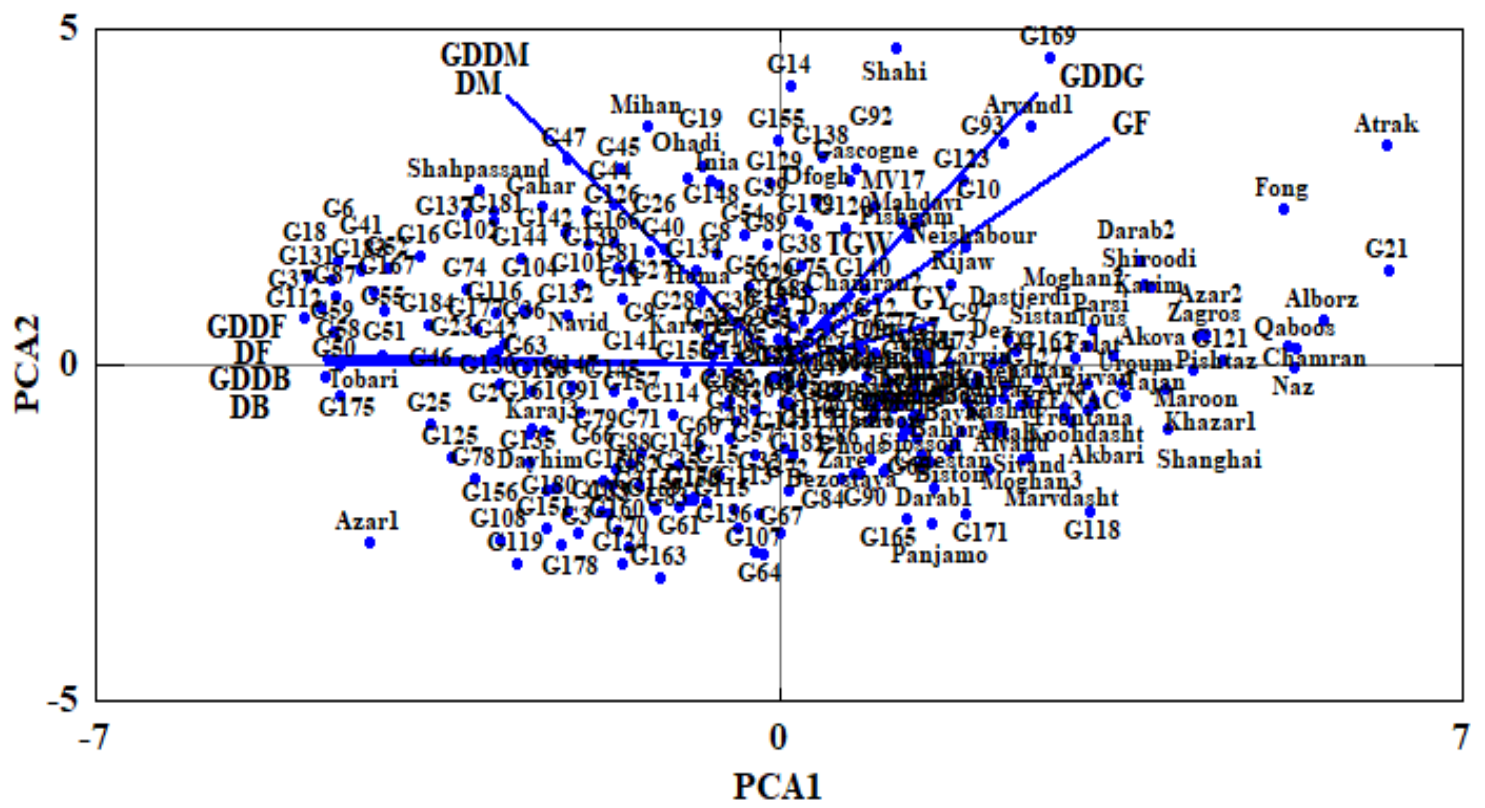

شكل 1- باى يلات تجزيه به مؤلفه هاى اصلى بر اساس صفات مورد مطالعه در (الف) سال اول و در (ب) سال دوم Figure 1. Principal component analysis biplot based on investigated traits in (A) first year and (B) second year 


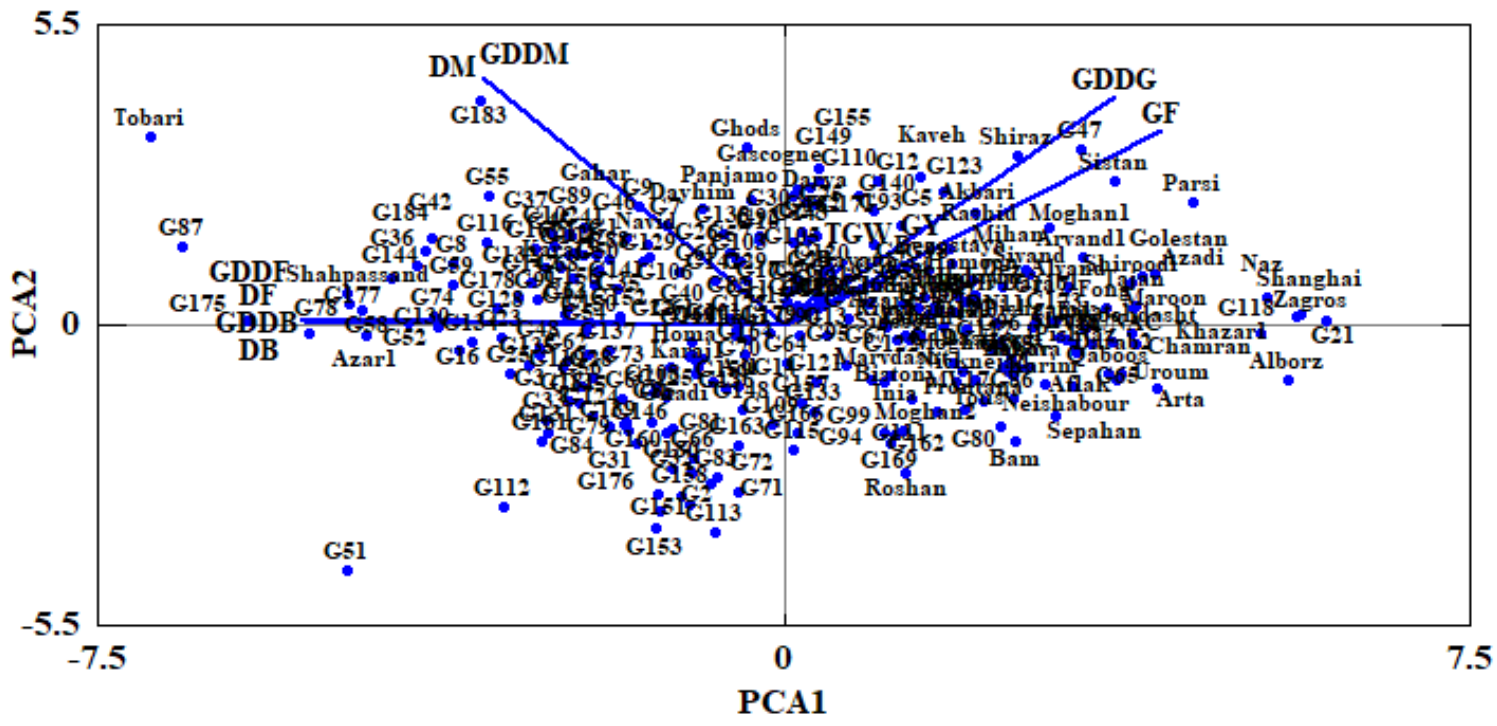

ادامه شكل ا- باى يالات تجزيه به مؤلفههاى اصلى بر اساس صفات مورد مطالعه در (الف) سال اول و در (ب) سال دوم Continue of Figure 1. Principal component analysis biplot based on investigated traits in (A) first year and (B) second year
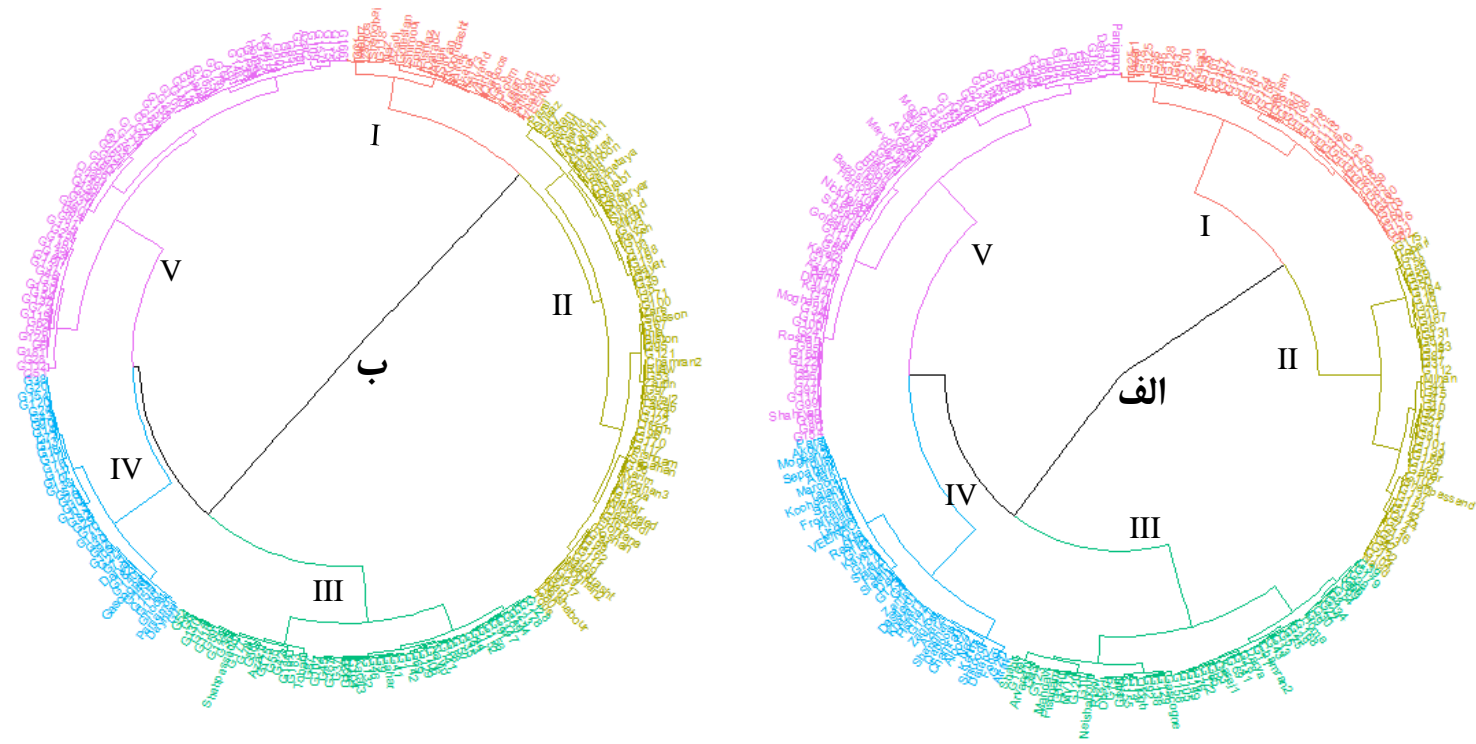

شكل ז- دندروكرام مربوط به كَروهبندى تودههاى بومى و ارقام زراعى گَندم با استفاده از صفات مورد مطالعه در (الف) سال اول و (ب) سال دوم

Figure 2. Classifying dendrogram in Iranian wheat landraces and cultivars based on investigated traits in (A) first year and (B) second year

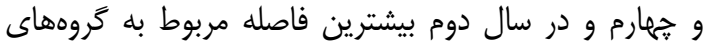

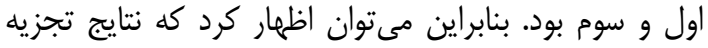

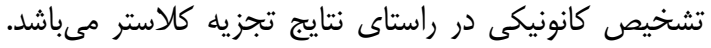

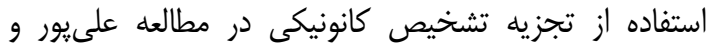

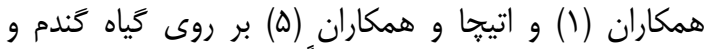

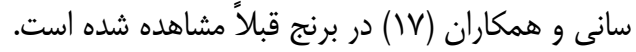

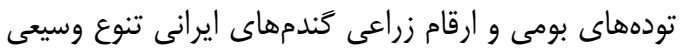

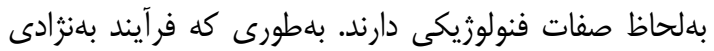

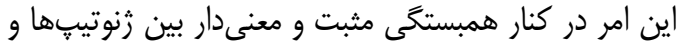

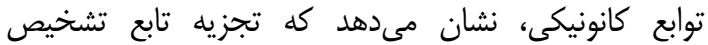

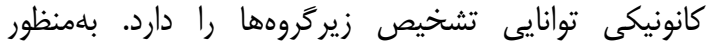

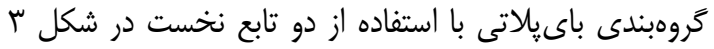

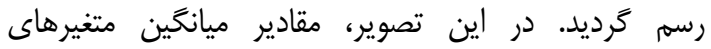

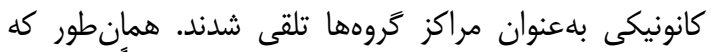
ملاحظه مى

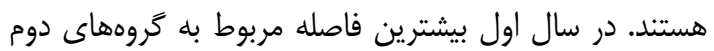


نمى گردد اما در ارقام زراعى كه عملكرد بيشترى در مقايسه

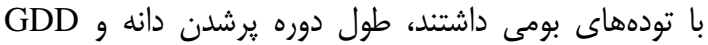

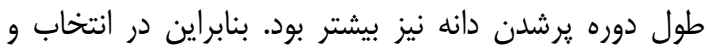

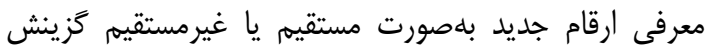

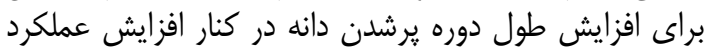

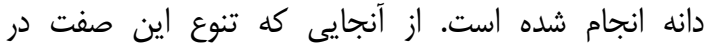

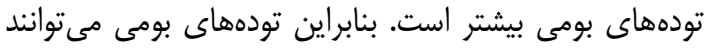
منبع ارزشمندى در راستاى انتخاب براى برائ تبردود اين صفت نيز

باشند.
كندم طى ساليان اخير در راستاى افزايش عملكرد و دوره

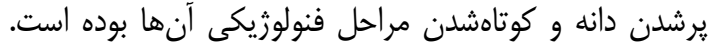

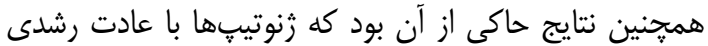

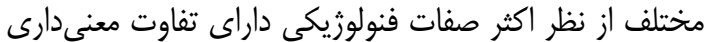

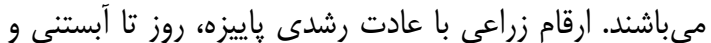

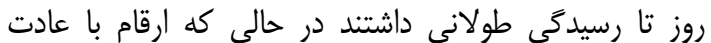

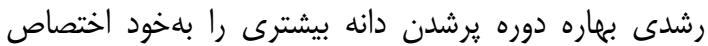

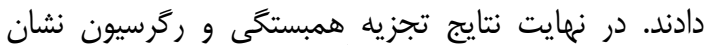
دادند كه GDD بيشتر الزاماً منجر به افزايش عملئ علكرد

جدول ع- تجزيه واريانس و مقايسه ميانگين صفات براى گروههاى حاصل از تجزيه خوشهاى طى دو سال زراعى Table 6. Analysis of variance and means comparison of studied traits for cluster analysis groups during two cropping

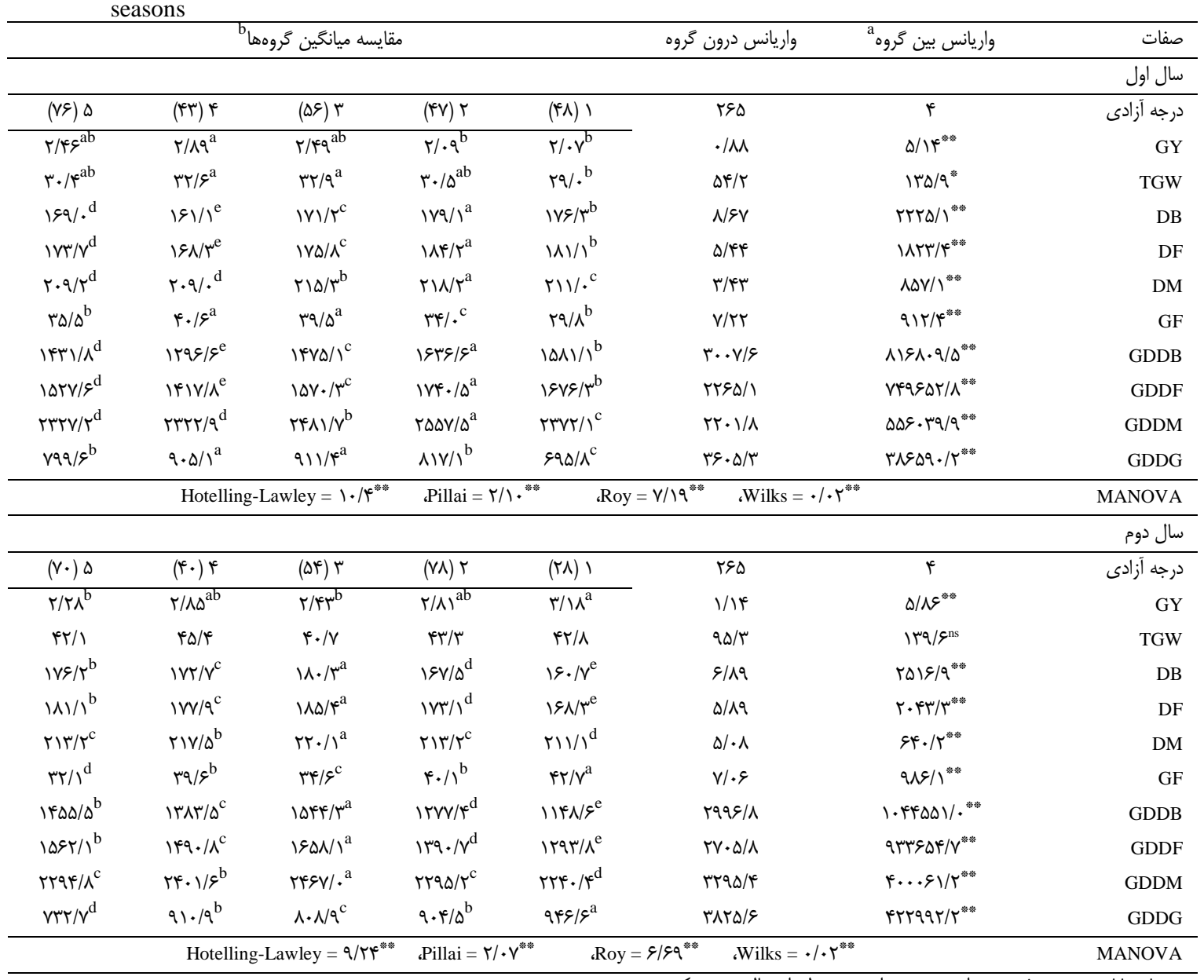

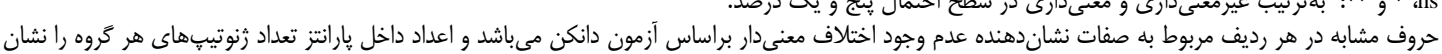

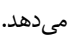

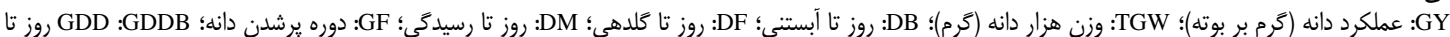

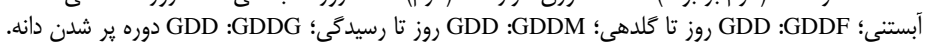


جدول V- تجزيه تابع تشخيص (اعتبارسنجى متقابل) براى صحت كرومبندى تجزيه خوشهاى (1) Table 7. Discriminant analysis (cross-validated) to grouping accuracy of cluster analysis

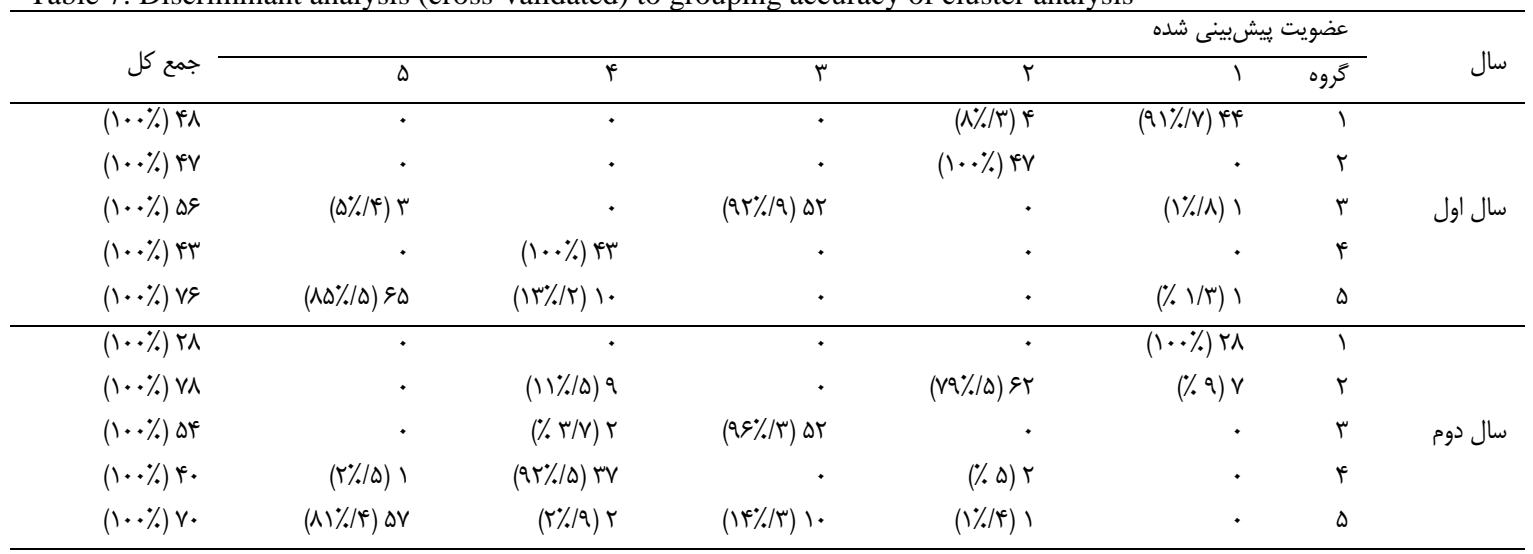

جدول ^- ماتريس ساختارى كانونيكى صفات بررسى شده در تودههاى بومى و ارقام زراعى كَندمهاى ايران Table 8. Canonical structure matrix of investigated traits in Iranian wheat landraces and cultivars

\begin{tabular}{|c|c|c|c|c|}
\hline \multicolumn{2}{|c|}{ سال دوم } & \multicolumn{2}{|c|}{ سال اول } & \multirow[b]{2}{*}{ صفات } \\
\hline تابع r & تابع 1 & تابع r & تابع 1 & \\
\hline.$/ 1 \% \omega^{*}$ &.$- / .9$. &.$/ \cdot \Delta T$ &.$- / .94$ & عملكرد دانه \\
\hline . $/ . r q$ & -./. & $.1 .9 \mathrm{~V}$ &.$- / \cdot T V$ & وزن هزار دانه \\
\hline.$- / 194$ & $\cdot / 9 \cdot r^{*}$ &.$- \cdot 1 \cdot v r$ & 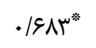 & روز تا آبستنى \\
\hline$-\cdot / 1 \cdot 1$ & $\cdot / \Lambda V^{* *}$ &.$- /$ IVe & $\cdot / 1 \cdot 9^{*}$ & روز تا كلدهى \\
\hline$\cdot /$ VGY" & $\cdot / F \mid F$ &.$|99|^{*}$ &.$|8|$. & روز تا رسيدگى \\
\hline$\cdot / N F D^{*}$ & $-\cdot /$ eq & . & $-\cdot / R \Lambda$. & دوره يرشدن دانه \\
\hline.$- / 148$ & $\cdot / M .^{*}$ &.$- / 1110$ & $\cdot / N \mid M^{*}$ & GDD روز تا آبستنى \\
\hline-.01 .9 & $\cdot \mid \Lambda \& \Lambda^{*}$ &.$- / 19$. & $\cdot / \Lambda \cdot r^{*}$ & GDD روز تا كلدهى \\
\hline$\cdot /$ MFr" & $\cdot / 4 \cdot 1$ & - & (4) & GDD روز تا رسيدگى \\
\hline$\cdot / V V q^{*}$ & $-\cdot / r \Delta T$ &. $\mid 991$ & $-\cdot / 1 \Delta \mathrm{V}$ & GDD دوره يرشدن دانه \\
\hline $1 / T V q$ & $9 / 891$ & $r / .9$. & $v / 19$. & مقادير ويزه \\
\hline$N \in / r$ & $V T / c$ & ^१/. & $99 / 1$ & درصد سهم تجمعى (\%) \\
\hline 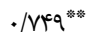 & 年 & $\cdot /$ Ar. ${ }^{*}{ }^{*}$ & $\cdot / 9 \mathrm{r}^{\text {**: }}$ & همبستخى كانونيكى \\
\hline
\end{tabular}
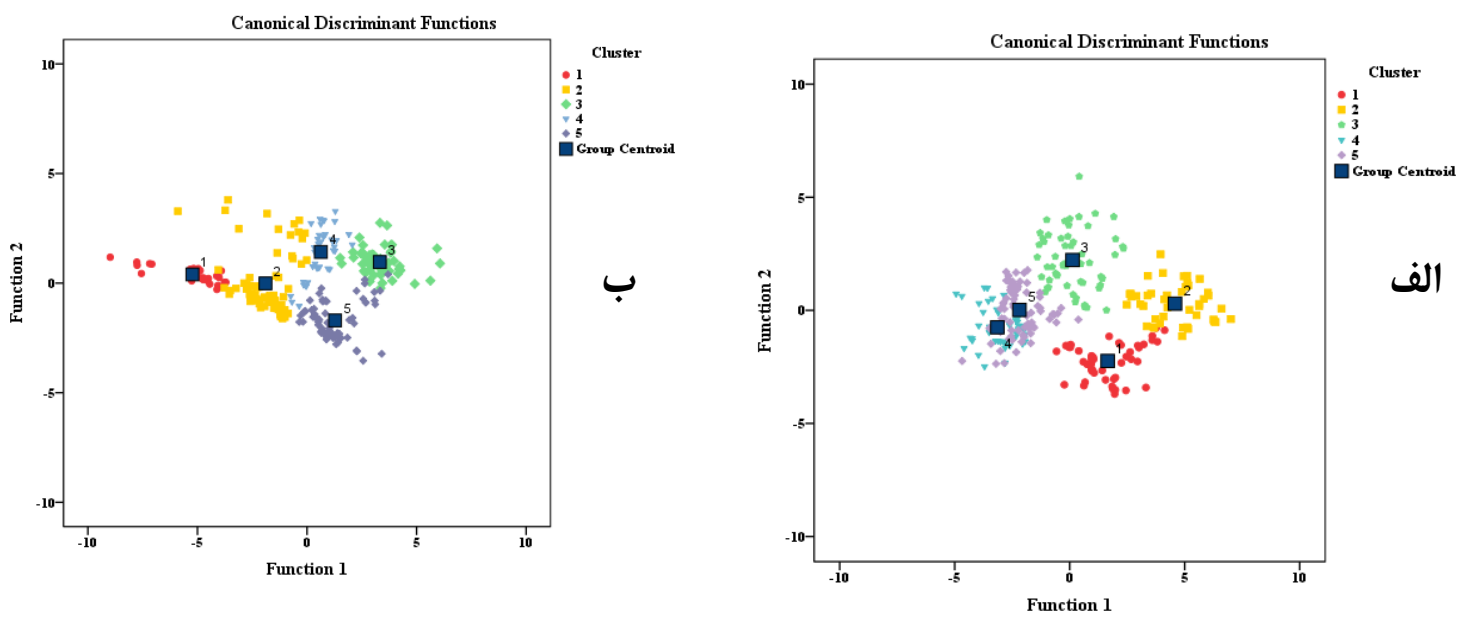

شكل س- گروهبندى ارقام زراعى و توده هاى بومى گندم مورد مطالعه براساس دو متغير كانونيك نخست در (الف) سال اول و در (ب) سال دوم Figure 3. Classification of studied wheat cultivars and landraces based on the first two canonical variables in (A) first year and (B) second year 
1. Alipour, H., M.R. Bihamta, V. Mohammadi and S.A. Peyghambari. 2017. Evaluation of genetic variability of agronomic traits in Iranian wheat landraces and cultivars. Journal of Crop Breeding, 9(22): 168-177 (In Persian).

2. Amini, A., A. Naderi and I. Lak zadeh. 2011. Investigation of phonological stages and grain yield of mid maturity wheat genotypes in response to different growth degree-day (GDD) accumulation in Ahvaz climate. The Quarterly Academic Journal of Crop Physiology, 3(10): 121-135 (In Persian).

3. Azizi, H. and B. Abdollahi Mandoulakani. 2015. Assessment of genetic variation in alfalfa (Medicago sativa L.) populations using Canonical Discriminant Analysis. Agronomy Journal (Pajouhesh and Sazandegi), 107: 183-189 (In Persian).

4. Darzi Ramandi, H., H. Najafi Zarini, V. Shariati, K, Razavi and S.K. Kazemitabar. 2018. Evaluation of the relationship between kernel size, phenological characteristics and grain yield of local wheat genotypes under water deficit stress conditions. Journal of Crop Breeding, 10(26): 76-94 (In Persian).

5. Eticha, F., G. Belay and E. Bekele. 2006. Species diversity in wheat landrace populations from two regions of Ethiopia. Genetic Resources and Crop Evolution, 53(2): 387-393.

6. FAO. 2018. Food and Agriculture Organization. Statistics: FAOSTAT agriculture. From http://fao.org/crop/statistics.

7. Jalal Kamali, M.R. and H.R. Sharifi. 2010. Variation in developmental stages and its relationship with yield and yield components of bread wheat cultivars under field conditions: II. Yield and its components. Seed and Plant Improvement Journal, 2-26(1): 1-23 (In Persian).

8. Jalal Kamali, M.R., H.R. Sharifi, M. Khodarahmi, R. Jokar, H. Torkaman and N. Ghavidel. 2007. Variation in developmental stages and its relationship with yield and yield components of bread wheat cultivars under field conditions: I. phenology. Seed and Plant Improvement Journal, 23(4): 445-472 (In Persian).

9. Koochaki, A. and M. Nasiri-mahalati. 1992. Crop ecology. First edition. Jahad Daneshgahi Press, Mashhad, (In Persian).

10. Lambert, A.M., A.J. Miller- Rushing and D.W. Inouye. 2010. Changes in snowmelt date and summer precipitation affect the flowering phenology of Erythronium grandiflorum (glacier lily; Liliaceae). American Journal of Botany, 97(9): 1431-1437.

11. Liakat Ali, M., A.M. McClung, M.H. Jia, J.A. Kimball, S.R. McCouch and C.E. Georgia. 2011. A rice diversity panel evaluated for genetic and agro-morphological diversity between subpopulations and its geographic distribution. Crop Science, 51(5): 2021-2035.

12. Miller, P., W. Lanier and S. Brandt. 2001. Using growing degree days to predict plant stages. Ag/Extension Communications Coordinator, Communications Services, Montana State University-Bozeman, Bozeman, MO, 1-2.

13. Mohammadi, S., D. Habibi, A. Kashani, F. Pakneihad, S. Bakhshipour and M.R. Ardakani. 2011. Study on physiological indices and agronomical characteristics of different Rice cultivars and plant spacing in West Mazandaran, Iran. Journal of Crop and Weed Ecophysiology, 5(3): 37-52 (In Persian).

14. Murphy, K.M., J.C. Dawson and S.S. Jones. 2008. Relationship among phenotypic growth traits, yield and weed suppression in spring wheat landraces and modern cultivars. Field Crops Research, 105(1-2): 107-115.

15. Naderi, A. 2013. Efficiency of heat unit and accumulative growing degree-day phonological stages and their relation with grain yield of wheat genotypes. Crop Physiology Journal, 5(18): 115-128 (In Persian).

16. Nasr Isfahani, M., Sh. Rafiezadeh, M. Rostampour, M.A. Karimkhah and S. Atashbahar. 2013. Evaluation of grain yield of promising wheat lines (Triticum aestivum) in Isfahan conditions. Crop Physiology Journal, 5(17): 31-48 (In Persian).

17. Sanni, K.A., I. Fawole, R.G. Guei, D.K. Ojo, E.A. Somado, D.D. Tia, S.A. Ogunbayo and I. Sanchez. 2008. Geographical patterns of phenotypic diversity in Oryza sativa landraces of Côte d'Ivoire. Euphytica, 160(3): 389-400.

18. Sharifi, H.R. 2016. Response of phenological development stages, grain yield and yield components of bread wheat cultivars with different growth habits to delayed planting. Seed and Plant Improvement Journal, 2-32(1): 21-44 (In Persian).

19. Shim, D., K.J. Lee and B.W. Lee. 2017. Response of phenology-and yield-related traits of maize to elevated temperature in a temperate region. The Crop Journal, 5(4): 305-316.

20. Slafer, G.A. 1994. Genetic improvement of field crops. CRC Press, 30: 488 pp.

21. Vafa, P., M. Barary, H. Darkhal and R. Naseri. 2014. Thermal requirement and the response of Corn hybrids (Zea mays L.) to different planting dates in Isfahan. Journal of Crop Ecophysiology, 8(2): 121-136 (In Persian).

22. Vaylay, R. and E. Van Santen. 2002. Application of canonical discriminant analysis for the assessment of genetic variation in tall fescue. Crop Science, 42(2): 534-539. 
23. Whittal, A., M. Kaviani, R. Graf, G. Humphreys and A. Navabi. 2018. Allelic variation of vernalization and photoperiod response genes in a diverse set of North American high latitude winter wheat genotypes. PloS One, 13(8): 1-17.

24. Wolde, G.M., C. Trautewig, M. Mascher and T. Schnurbusch. 2019. Genetic insights into morphometric inflorescence traits of wheat. Theoretical and Applied Genetics, 132(6): 1661-1676.

25. Yousefi Moghaddam, R., S. Khoramdel, M. Bannayan Aval and M. Nassiri Mahallati. 2018. Comparison of old and new dryland wheat cultivars in response to different planting dates. Applied Research in Field Crops, 31(2): 46-72 (In Persian).

26. Zafarian, E., A. Ebrahimi, A. Abbasi and E. Asadi. 2019. Required growing degree-days (GDDs) for each phenological stage of Fritillaria imperialis. Journal of Rangeland Science, 9(1): 62-73.

27. Zarebayati, A., M. Khodarahmi and K. Mostafavi. 2017. Relationship among traits and path analysis for grain yield of winter wheat cultivars under normal and drought stress conditions. Journal of Agronomy and Plant Breeding, 13(4): 85-95 (In Persian). 


\title{
Assessment of Growing Degree-Days Values of Phenological Stages in some Iranian Bread Wheat Cultivars and Landraces
}

\author{
Hadi Alipour ${ }^{1}$, Hossein Abdi ${ }^{2}$ and Mohammad Reza Bihamta ${ }^{3}$ \\ 1- Assistant Professor, Department of Plant Production and Genetics, Urmia University, Urmia, \\ (Corresponding author: ha.alipour@urmia.ac.ir) \\ 2- Ph.D. Candidate, Department of Plant Production and Genetics, Urmia University, Urmia \\ 3- Professor, Department of Agronomy and Plant Breeding, University of Tehran, Karaj \\ Received: August 11, $2019 \quad$ Accepted: January 7, 2020
}

\begin{abstract}
Growing degree-days (GDD) is considered as an alternative indicator for counting calendar days in studying of crop phenology stages. In the current study, genetic diversity of 86 Iranian wheat cultivars and 184 landraces were investiagted based on the phenological stages (day to booting, day to flowering, day to maturity and GDD of these stages) along with grain filling period, thousand grain weight and grain yield during 2013-2014 growing seasons in augmented design with 3 control cultivars (Azar, Navid and Pishtaz) in 6 incomplete blocks in Agronomy and Plant Breeding research filed, University of Tehran. The results illustrated that there is a significant difference between cultivars and landraces, so that the cultivars had higher yield and less grain filling period than the landraces. On the other hand, the results indicated that the genotypes with different growth habits have a significant difference in terms of most of the phenological traits which it could be useful either in directly or indirectly selection for increasing the length of the grain filling period along with grain yield. Since the diversity of phenological traits were higher in landraces, they can be a valuable source for selection to improving these traits. Finally, due to the importance of the phenological stages and GDD index, it is recommended that these traits also consider as well as yield and yield components in order to obtain more accurate results.
\end{abstract}

Keywords: Cultivars, GDD, Genetic diversity, Landraces, Thermal need 